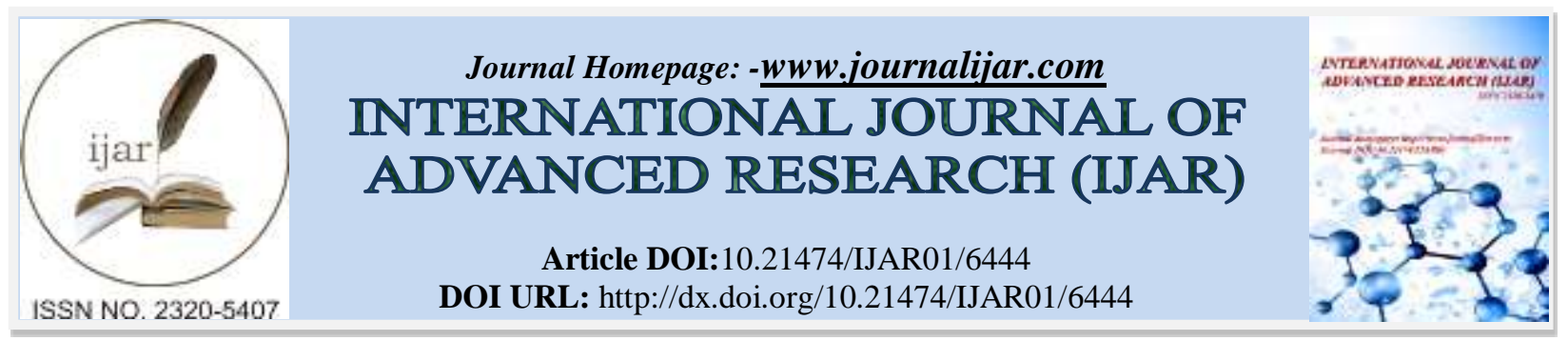

RESEARCH ARTICLE

\title{
PLACENTAL MESENCHYMAL DYSPLASIA: REPORT OF A CASE AND REVIEW OF THE LITERATURE.
}

\author{
Stefania Erra, Alessia Frigeri and Alessia Picozzi. \\ Surgical pathology department, Santo Spirito Hospital, Casale Monferrato (Italy).
}

\section{Manuscript Info}

Abstract

Manuscript History

Received: 04 December 2017

Final Accepted: 06 January 2018

Published: February 2018

Copy Right, IJAR, 2018,. All rights reserved.

\section{Introduction:-}

The PMD is a rare vascular abnormality of the placenta, with incidence of $0,02 \%$. Macroscopically, placenta is macrosomic and thickened, with dilated sub-chorionic vessels and anomalous anastomoses. Placental weight can be over $1000 \mathrm{gr}$ at the end of the pregnancy.

This pathology was first reported by Moscoso in 1991 (1). He described an enlarged placenta with widespead villus hyperplasia of the peduncle and increased levels of $\alpha$-fetoprotein in blood. Vessels on the fetal plate showed aneurysmal and varicose dilatations, and the stem villi appeared as semitranslucent lobulated structures. At microscopy level, this pathologic placenta presented excessive proliferation of mesenchymatous tissue with foci of myxoid degeneration with none pathological trophoblastic proliferation .

Placenta with PMD morphology can have a normal karyotype or it may be associated to chromosomal abnormality. In $23 \%$ of cases, PMD is linked to Beckwith-Wiedemann Syndrome(BWS), suggesting a common genetic alterations (2).

It has been described a spectrum of phenotypic variations in PMD, sometimes limited only to the placenta, many times involving both fetus and placenta. In these last cases, abnormal expression of one or more genes on chromosome 11 has been observed, above all in the region 11p15.5 (3).

The PMD diagnostic suspect is based on detection of ultrasound with diffuse cystic aspect of the placenta, with image suggested for molar degeneration (80\% of cases) and placentomegalia (50\% of cases).

Placental mesenchymal dysplasia is associated with intrauterine growth restriction or intrauterine fetal death in the third trimester of gestation, even in absence of fetal malformations.

\section{Case Report:-}

Placenta at the 30th week of pregnancy of a first child 30 year old indian woman was send in surgical pathology department for macroscopic aberrant vascular alterations and high weight, precisely 1700 gr (fig 1). It presented a segment of umbilical cord with velamentous insertion. The chorionic plate, size $23 \times 22 \times 7 \mathrm{~cm}$, was characterized by sub-chorionic vessels markedly swollen and arborescent aspect, anastomosed in a tangle of subverted and unrecognizable structures similar to cysts (fig 2,3). 
Microscopically, the chorionic plate presented thinning of the terminal villi, presence of large caliber stem ones, with fibrotic and edematous stroma, marked dilatation of the vessels with hamartomatous appearance (fig.4,5). There were also areas of hemorrhagic and ischemic infarction associated with trombotic occlusion of some structures. In addition, acute granulocytic inflammation was found on decidua and funiculus.

At birth, the female newborn weighed 2400 grams and had no obvious malformations. She was admitted to neonatal intensive care for presumably respiratory problems related to funisitis.

No genetic examination was performed on the placenta and the newborn for economic reasons, since the mother was unable to bear the costs of such diagnostic investigations.

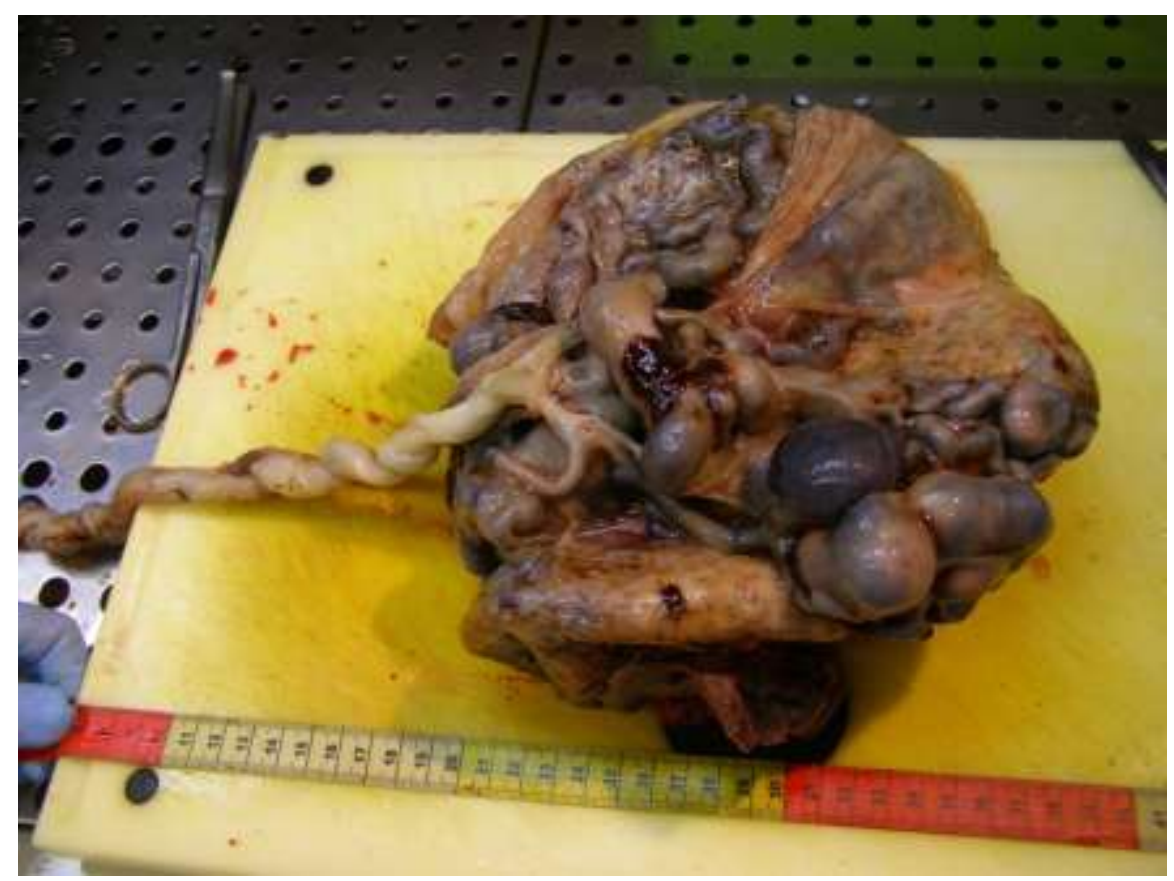

Fig. 1 :- placenta specimen with placentomegaly

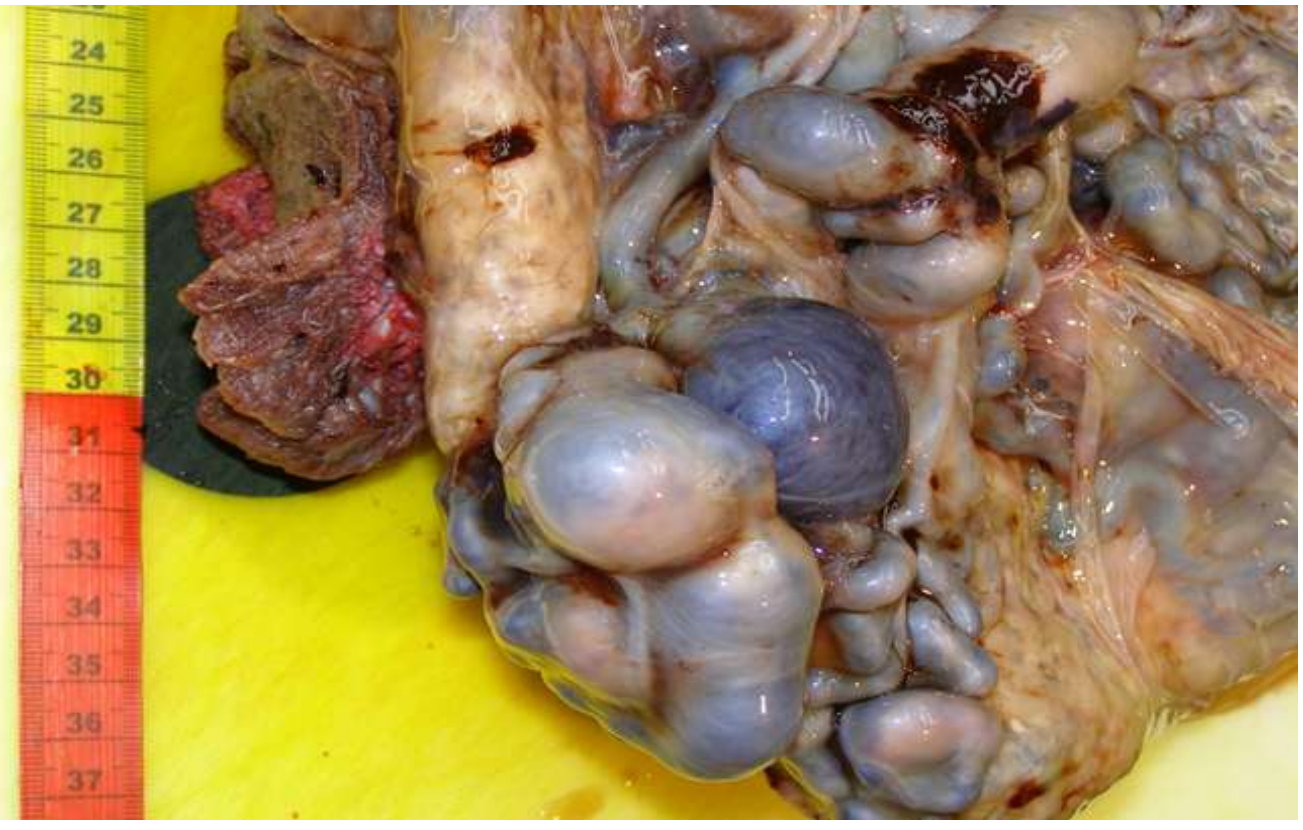


Fig 2:- detailed gross vessels of placenta

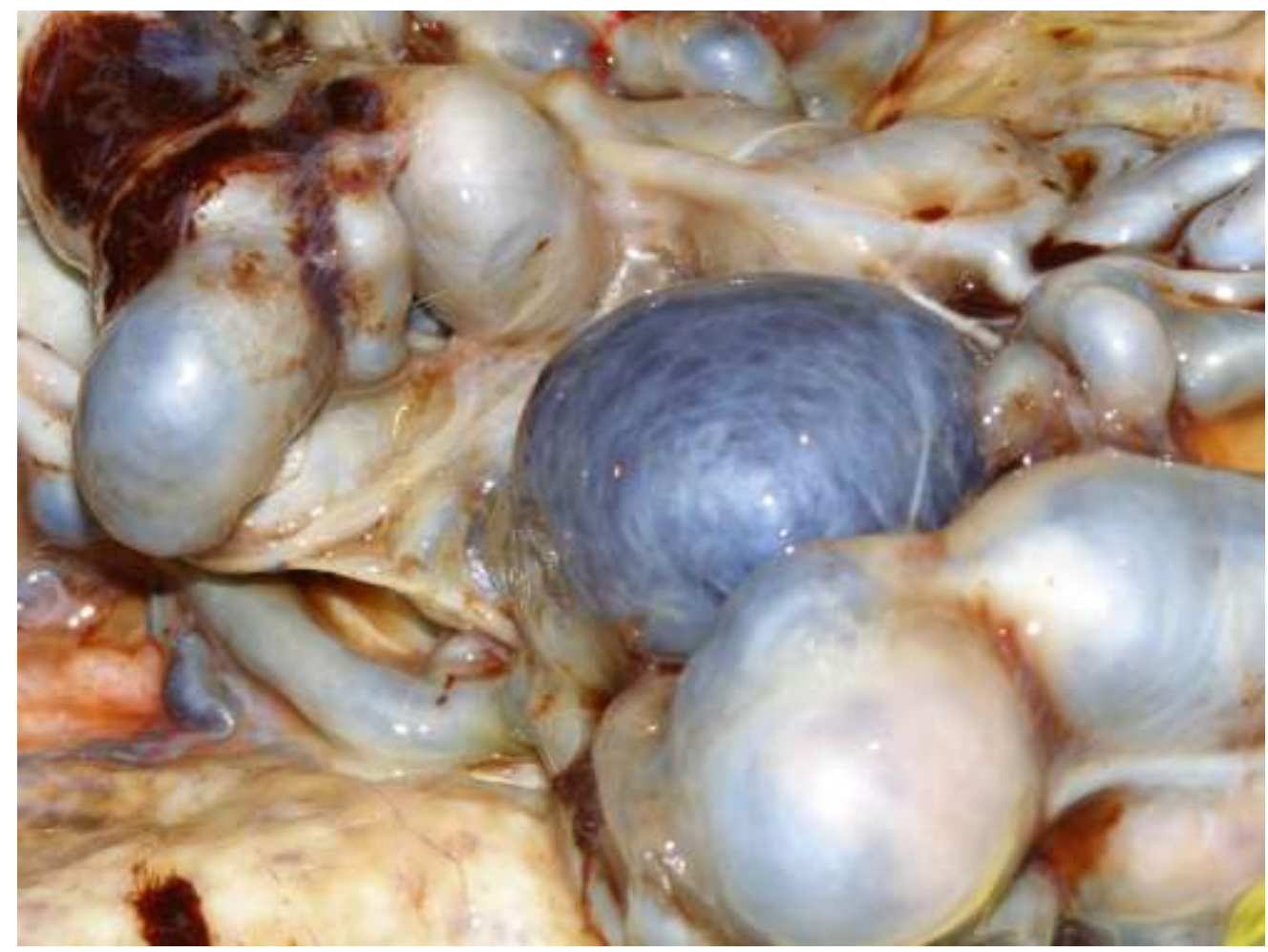

Fig. 3:- cyst-like aspect od sub-decidual placental vessels

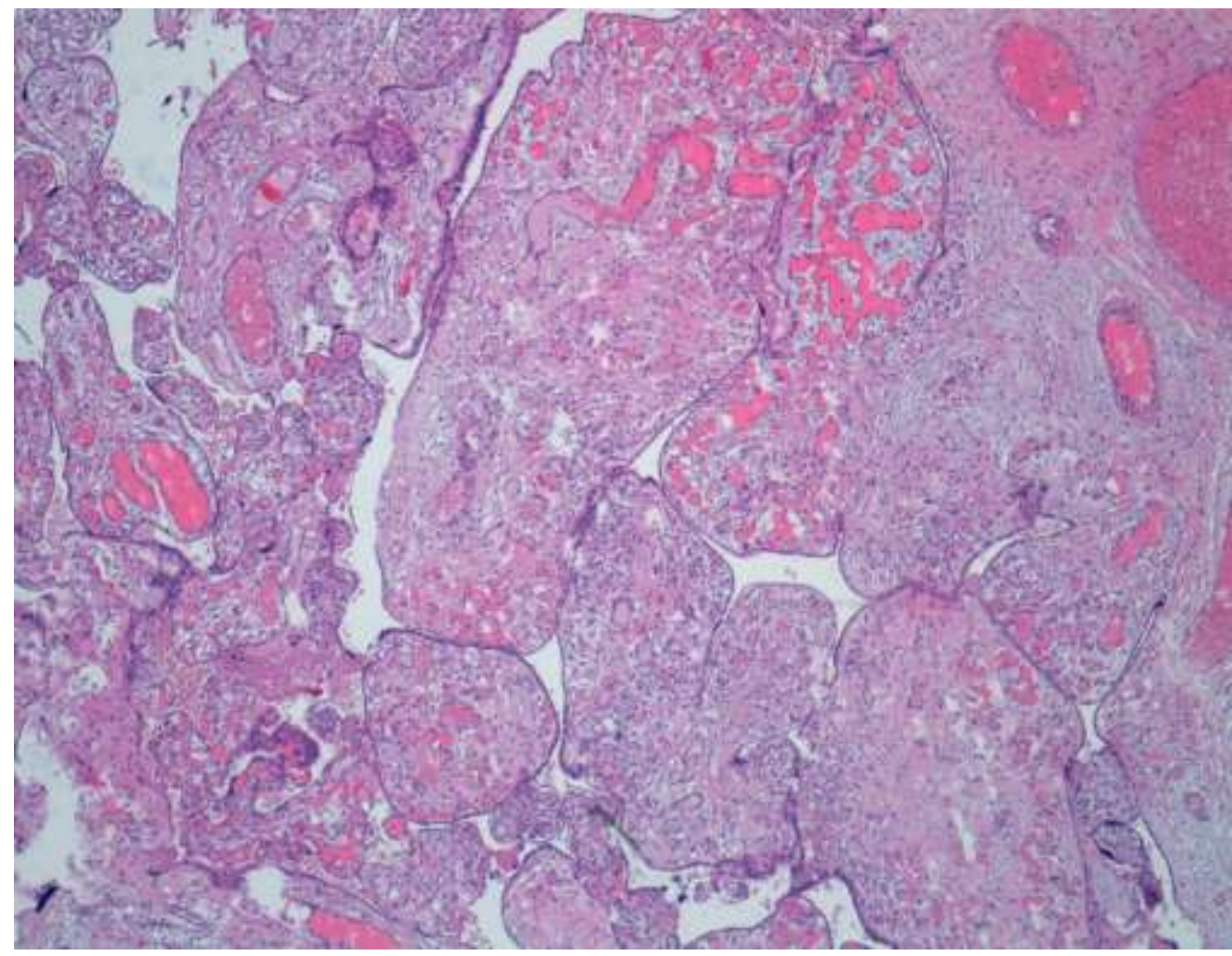

Fig.4:- E.E slide with gross anomalous stem villi (4x) 


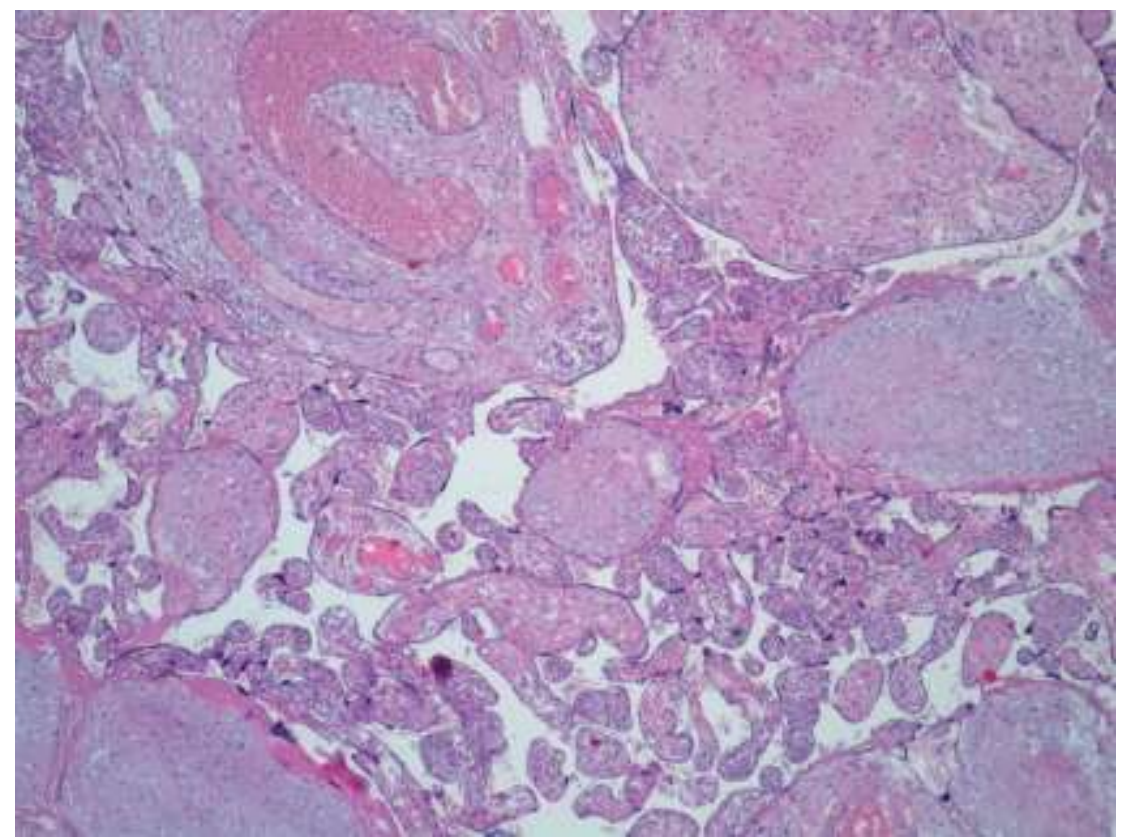

Fig. 5:- E.E. slide representing abnormal structure of chorionic plate without well-formed intermediate and terminal villi

\section{Discussion:-}

PMD is a rare condition linked to chromosomal aberrations. The $33 \%$ of fetus with PMD is affected by Intra Uterine Growth Retard (IUGR), the $38 \%$ by fetal malformations and the $13 \%$ by fetal stillbirth in extreme cases (4,5). In $9 \%$ of cases maternal complications have been reported : preeclampsia (single or in combination, presence of edema, proteinuria, hypertension), eclampsia with gestational hypertension, HELLP Syndrome (hemolysis, elevated liver enzymes, thrombocytopenia). Finally, in 23\% of cases the PMD is associated with Beckwith-Wiedemann Syndrome, suggesting a common genetic bases involving the abnormal expression of one or more genes on chromosome 11. As regards the origin of this placental anomaly, one of the most reliable hypothesis suggests that PMD occurs when 1 ovum is fertilized by 2 spermatozoa with errors during the first meiotic division of the fertilized egg cell (5). These errors could be responsible of silencing or disruption of the imprinted genes that regulate normal placental formation, with resulting in aberrant growth of placental tissue and placentomegaly (6).

Only 110 cases of PMD have been reported in literature in an article dated 2014 (7), while not more than 10 cases have been described after this date. Despite everything, the mesenchymal dysplasia of the placenta constitutes an underestimated diagnostic entity, because early cases can be confused with partial hydatidiform mole, above all in abortions of the first trimester of gestation for the similar sonographic findings of the two entities.

The vesicular mole is characterized by the presence of swelling and abnormal stem villi, with trophoblastic alterations and without the net of little vessels normally located in villous stroma. Hydatidiform mole is a gestational trophoblastic disease linked to chromosomal aberrations of the product of conception and incompatible with the life of the fetus. It becomes important the correct differential diagnosis between these two entities with similar morphology but different outcomes. In fact, in PMD there usually can be observed caryotypically and morphological normal fetus and a good maternal-fetal outcome (8), as in the present reported case. An exception is represented by the relatively frequent presence of BWS associated with PMD, which can be recognized through study of the karyotype with CGH-Array $(2,3)$.

The BWS is a pan-ethnic disease, with an estimated prevalence of 1 out of 13700; the incidence is equal in both sexes. Patients affected by BWS show overgrowth in the second half of pregnancy and in the first years of life; in addition to this it can be observed macrosomia, macroglossia, hemihyperthrophy, omphalocele, embryonic tumors, visceromegaly, cleft palate and cardiomyopathy. 
The BWS is due to genetic and/or epigenetic alterations that modify the function genes undergo imprinting on chromosome 11p15.5. The disease is sporadic in $85 \%$ of cases, while in other cases there is a familiar transmission. Both the PMD and the BWS are related to the abnormal expression of one or more genes on chromosome 11. In the light of what is reported, it is not explained how the presence of a chromosomal alteration established at the beginning of pregnancy can determine PMD without repercussions on the fetus. Most cases of PMD are, in fact, described in fetuses of the last trimester of gestation without chromosomal alterations. In such cases endouterine death or IUGR are caused by vascular insufficiency of the placenta with PMD.

\section{Conclusion:-}

PMD is a rare disease affecting placenta and it is linked to mutations of chromosome 11. Most of cases are reported in cariotypically normal fetuses, with IUGR or fetal intrauterine death. The most important differential diagnosis must be made with the molar pathology of the trophoblast. In fact, hydatiforme mole represents a gestational trophoblastic disease due to chromosomal aberrations affecting fetus too. This differential diagnosis can't be made only on the basis of clinical and ultrasound data, so pathologists make the correct diagnosis on the placenta after the birth. For this reason it is essential to consider that the PMD can have morphological characteristics that are not always pathognomonic, even if peculiar. Consequently, it is important that pathologists take this diagnostic entity into consideration in the presence of a placenta with a weight much higher than normal and with an abnormal vascularization, consisting of dilateted and arborescent vessels, sometimes of a cyst-like appearance.

In case of suspected diagnosis it can be performed fetal karyotype, $\alpha$-fetoprotein dosage, careful study to search for signs of BWS and in particular to exclude liver tumors, serial ultrasounds and histological examination of the placenta. Many children with PMD don't suffer of malformative defects, but the prognosis poses the risk of early death from secondary complications to hypoglycemia, prematurity or cardiomyopathy. In patients who survive infancy, the prognosis is generally good.

In PMD genetic counseling is always recommended, even if a hereditary transmission of the chromosomal defect has not been described.

Finally we need to remember the usefulness of a comprehensive macroscopic sampling of placenta in case of suspected mesenchymal dysplasia and the possibility of using ancillary methods to histology. About that immunohistochemical research of p57 protein expression represents a simple but important one, because in stromal cells of dysplastic stem villi in all cases of PMD a loss of p57 expression can be detected $(9,10)$.

\section{References:-}

1. Moscoso G, Jauniaux E, Hustin J Placental vascular anomaly with diffuse mesenchymal stem villous hyperplasia. A new clinic-pathological entity? Pathol Res Pract 1991; 187: 324328.

2. H'mida D, Gribaa M, Yacoubi T, et al. Placental mesenchymal dysplasia with Beck-Wiedemann syndrome fetus in the context of biparental and androgenic cell lines. Placenta. 2008;29(5):454-460.

3. Robinson WP, Slee J, Smith N, et al. Placental mesenchymal dysplasia associated with fetal overgrowth and mosaic deletion of the maternal copy of 11p15.5. Am J Med Genet A. 2007;143A(15):1752-1759

4. Pham T, Steele J, Stayboldt C, Chan L, Benirschke K. Placental mesenchymal dysplasia is associated with high rates of intrauterine growth restrictions and fetal demise: a report of 11 new cases and a review of the literature. Am J Clin Pathol. 2006; 126(1):67-78.

5. Nogba Pawoo, DO; Debra S. Heller, MD Placental Mesenchymal Dysplasia Arch Pathol Lab Med. 2014;138:1247-1249; doi: 10.5858/arpa.2013-0399-RS

6. Surti U1, Yatsenko S2, Hu J3, Bellissimo D3, Parks WT4, Hoffner L5. Maternal GRB10 microdeletion is a novel cause of cystic placenta: Spectrum of genomic changes in the etiology of enlarged cystic placenta. Placenta. 2017 Sep;57:33-41. doi: 10.1016/j.placenta.2017.05.018. Epub 2017 Jun 2.

7. Marcello Pecoraro Toscano, Regina Schultz Placental mesenchymal dysplasia: case report with gross and histological findings Autopsy and Case Reports 2014;4(4):51-6 http://dx.doi.org/10.4322/acr.2014.039

8. Gizzo S, Di Gangi S, Patrelli TS, Saccardi C, D’Antona D, Nardelli GB. Placental mesenchymal dysplasia: Can early diagnosis ensure a good materno-foetal outcome? A case report. Arch Gynecol Obstet. 2012;286:157. 
9. Allias F1, Lebreton F, Collardeau-Frachon S, Azziza J, Pasquier CJ, Arcin-Thoury F, Patrier S, DevouassouxShisheboran M. Immunohistochemical expression of p57 in placental vascular proliferative disorders of preterm and term placentas. Fetal Pediatr Pathol. 2009;28(1):9-23. doi: 10.1080/15513810802545350

10. Chilosi M1, Piazzola E, Lestani M, Benedetti A, Guasparri I, Granchelli G, Aldovini D, Leonardi E, Pizzolo G, Doglioni C, Menestrina F, Mariuzzi GM. Differential expression of p57kip2, a maternally imprinted cdk inhibitor, in normal human placenta and gestational trophoblastic disease. Lab Invest. 1998 Mar;78(3):269-76 